\title{
Levitin-Polyak Well-Posedness of an Equilibrium-Like Problem in Banach Spaces
}

\author{
Ru-liang Deng \\ College of Economic Mathematics, Southwestern University of Finance and Economics, Chengdu, Sichuan 611130, China \\ Correspondence should be addressed to Ru-liang Deng; dengrl@swufe.edu.cn
}

Received 8 February 2014; Accepted 26 April 2014; Published 21 May 2014

Academic Editor: Qing-bang Zhang

Copyright (c) $2014 \mathrm{Ru}$-liang Deng. This is an open access article distributed under the Creative Commons Attribution License, which permits unrestricted use, distribution, and reproduction in any medium, provided the original work is properly cited.

The concept of Levitin-Polyak well-posedness of an equilibrium-like problem in Banach spaces is introduced. Under suitable conditions, some characterizations of its Levitin-Polyak well-posedness are established. Some conditions under which an equilibrium-like problem in Banach spaces is Levitin-Polyak well-posed are also derived.

\section{Introduction}

In 1966, Tykhonov [1] first established the well-posedness of a minimization problem, which has been known as Tykhonov well-posedness. Since it is important in optimization problems, various concepts of well-posedness have been introduced and studied in past decades. For more about the well-posedness, we refer to [2-4] and the references therein.

The Tykhonov well-posedness of a constrained minimization problem requires that every minimizing sequence should lie in the constraint set. In many situations, the minimizing sequence produced by a numerical optimization method usually fails to be feasible but gets closer and closer to the constraint set. Levitin and Polyak [5] generalized the concept of Tykhnov well-posedness by requiring the existence and uniqueness of minimizer and the convergence of every generalized minimizing sequence toward the unique minimizer, which has been known as Levitin and Polyak wellposedness. There are a lot of results concerned with Tykhonov well-posedness, LP well-posedness, and their generalizations for minimization problems. For details, we refer to [1-3, 5-7].

Recently, the concept of well-posedness has been extended to many other fields, including Nash equilibrium
[8], inclusion problems, and fixed point problems [9-13]. Lemaire $[12,13]$ studied the relations between the wellposedness of minimization problems, inclusion problems, and fixed point problems. Fang et al. [11] proved that the well-posedness of a general mixed variational inequality is equivalent to the existence and the uniqueness of its solution in the Hilbert space. Recently, Ceng and Yao [9] got some results for the well-posedness of the generalized mixed variational inequality, the corresponding inclusion problem, and the corresponding fixed point problem. On the other hand, Li and Xia [14] considered the Levitin-Polyak well-posedness of a generalized variational inequality in Banach space. And they showed that the Levitin-Polyak well-posedness of a generalized variational inequality is equivalent to the uniqueness and existence of its solutions. However, there has been no result for the Levitin-Polyak well-posedness of an equilibrium-like problem.

Motivated and inspired by the research work going on in this field, in this paper, we extend the notion of Levitin-Polyak well-posedness to an equilibrium-like problem in Banach spaces and give some metric characterizations of its LevitinPolyak well-posedness. Finally, we derive some conditions under which an equilibrium-like problem is Levitin-Polyak well-posed. 


\section{Preliminaries}

Let $X$ be a real reflexive Banach space with its dual $X^{*}$ and let $K$ be a nonempty, closed, and convex subset of $X$. Let $F$ : $X \rightarrow 2^{X^{*}}$ be a set-valued mapping, and let $\phi: X^{*} \times X \times X \rightarrow$ $\mathbb{R}$ be a functional. In this paper, we consider the following equilibrium-like problem associated with $(F, \phi, K)$ :

$\operatorname{ELP}(F, \phi, K)$ : find $x \in K$ such that for some $u \in F(x)$,

$$
\phi(u, x, y) \leq 0, \quad \forall y \in K .
$$

Definition 1. Let $A, B$ be nonempty subsets of $X$. The Hausdorff metric $\mathscr{H}(\cdot, \cdot)$ between $A$ and $B$ is defined by

$$
\mathscr{H}(A, B)=\max \{e(A, B), e(B, A)\},
$$

where $e(A, B)=\sup _{a \in A} d(a, B)$ with $d(a, B)=\inf _{b \in B}\|a-b\|$.

Lemma 2 (Nadler's theorem [7]). Let $(X,\|\cdot\|)$ be a normed vector space and let $\mathscr{H}(\cdot, \cdot)$ be the Hausdorff metric on the collection $C B(X)$ of all nonempty, closed, and bounded subsets of $X$, induced by a metric $d$ in terms of $d(u, v)=\|u-v\|$, which is defined by $\mathscr{H}(U, V)=\max \{e(U, V), e(V, U)\}$, for $U$ and $V$ in $C B(X)$, where $e(U, V)=\sup _{x \in U} d(x, V)$ with $d(x, V)=\inf _{y \in V}\|x-y\|$. If $U$ and $V$ lie in $C B(X)$, then, for any $\epsilon>0$ and any $u \in U$, there exists $v \in V$ such that $\|u-v\| \leq(1+\epsilon) \mathscr{H}(U, V)$. In particular, whenever $U$ and $V$ are compact subsets in $X$, one has $\|u-v\| \leq \mathscr{H}(U, V)$.

Definition 3 (see [9]). A nonempty set-valued mapping $F$ : $X \rightarrow 2^{X^{*}}$ is said to be

(i) $\mathscr{H}$-hemicontinuous if, for any $x, y \in X$, the function $t \mapsto \mathscr{H}(F(x+t(y-x), F(x)))$ from $[0,1]$ into $\mathbb{R}^{+}=[0,+\infty)$ is continuous at $0^{+}$, where $\mathscr{H}(\cdot, \cdot)$ is the Hausdorff metric defined on $C B(X)$;

(ii) $\mathscr{H}$-uniformly continuous if, for all $\epsilon>0$, there exists $\delta>0$ such that for all $x, y \in X$ with $\|x-y\|<\delta$, one has $\mathscr{H}(F(x), F(y))<\epsilon$, where $\mathscr{H}(\cdot, \cdot)$ is the Hausdorff metric defined on $C B(X)$.

Definition 4. Let $X$ and $Y$ be two topological spaces and $x \in X$. A set-valued mapping $F: X \rightarrow 2^{Y}$ is said to be upper semicontinuous (u.s.c. in short) at $x$, if for any neighbourhood $V$ of $F(x)$, there exists a neighbourhood $U$ of $x$ such that $F(y) \subset V$, for all $y \in U$. If $F$ is u.s.c. at each point of $X$, we say that $F$ is u.s.c. on $X$.

Definition 5 (see [15]). Let $A$ be a nonempty subset of $X$. The measure of noncompactness $\mu$ of the set $A$ is defined by

$$
\begin{gathered}
\mu(A)=\inf \{\epsilon>0: \\
A \subset \bigcup_{i=1}^{n} A_{i}, \operatorname{diam} A_{i}<\epsilon, \\
i=1,2, \ldots, n\},
\end{gathered}
$$

where $\operatorname{diam} A_{i}$ denotes the diameter of the set $A_{i}$, for $i=$ $1,2, \ldots, n$.

Definition 6. Let $X$ be a real reflexive Banach space with its dual $X^{*}$ and let $F: X \rightarrow 2^{X^{*}}$ be a set-valued mapping. A functional $\phi: X^{*} \times X \times X \rightarrow \mathbb{R}$ is said to be monotone with respect to $F$, if for any $x, y \in X$ and $u \in F(x), v \in F(y)$, $\phi(u, x, y) \geq \phi(v, x, y)$.

Remark 7. If $\phi(u, x, y)=\langle u, x-y\rangle$, for all $x, y \in X$ and $u \in F(x)$, it is easy to know that $\phi$ is monotone with respect to $F$ which reduces to $F$ being monotone.

We first prove the following proposition.

Proposition 8. Let $K$ be a nonempty, closed, and convex subset of $X$ and let $F: X \rightarrow 2^{X^{*}}$ be a nonempty compact-valued mapping which is $\mathscr{H}$-hemicontinuous. Let $\phi: X^{*} \times X \times X \rightarrow \mathbb{R}$ be monotone with respect to $F$, continuous in first argument, and concave in third argument. Moreover, $\phi(u, x, x)=0$, for all $u \in X^{*}, x \in K$. Then, for a given $x \in K$, the following statements are equivalent:

(i) there exists $u \in F(x)$ such that $\phi(u, x, y) \leq 0$, for all $y \in K$;

(ii) $\phi(v, x, y) \leq 0$, for all $y \in K, v \in F(y)$.

Proof. First, we assume that for some $u \in F(x), \phi(u, x, y) \leq 0$, for all $y \in K$. Because $\phi$ is monotone with respect to $F$, we have

$$
\phi(v, x, y) \leq 0, \quad \forall y \in K, \quad v \in F(y) .
$$

Conversely, suppose that for all $y \in K, v \in F(y)$, we obtain

$$
\phi(v, x, y) \leq 0
$$

For any given $y \in K$, we define $y_{t}=t y+(1-t) x$ for all $t \in(0,1)$. Replacing $y$ by $y_{t}$ in the left-hand side of the last inequality, we have that, for each $v_{t} \in F\left(y_{t}\right)$,

$$
\begin{aligned}
0 & \geq \phi\left(v_{t}, x, y_{t}\right) \\
& =\phi\left(v_{t}, x, t y+(1-t) x\right) \\
& \geq t \phi\left(v_{t}, x, y\right)+(1-t) \phi\left(v_{t}, x, x\right) \\
& =t \phi\left(v_{t}, x, y\right) .
\end{aligned}
$$

This implies that

$$
\phi\left(v_{t}, x, y\right) \leq 0, \quad \forall v_{t} \in F\left(y_{t}\right), t \in(0,1) .
$$


Since $F: X \rightarrow 2^{X^{*}}$ is a nonempty compact-valued mapping, $F\left(y_{t}\right)$ and $F(x)$ are nonempty compact and hence lie in $C B(X)$. From Lemma 2, we get that, for each $t \in(0,1)$ and for each fixed $v_{t} \in F\left(y_{t}\right)$, there exists a $u_{t} \in F(x)$ such that

$$
\left\|v_{t}-u_{t}\right\| \leq(1+t) \mathscr{H}\left(F\left(y_{t}\right), F(x)\right) .
$$

Since $F(x)$ is compact, without loss of generality, we assume that $u_{t} \rightarrow u \in F(x)$ as $t \rightarrow 0^{+}$. Since $F$ is $\mathscr{H}$ hemicontinuous, we get that as $t \rightarrow 0^{+}$,

$$
\left\|v_{t}-u_{t}\right\| \leq(1+t) \mathscr{H}\left(F\left(y_{t}\right), F(x)\right) \longrightarrow 0
$$

This implies that $v_{t} \rightarrow u \in F(x)$ as $t \rightarrow 0^{+}$. Since $\phi$ is continuous in first argument, by $(*)$ we obtain that there exists an $u \in F(x)$ such that

$$
\phi(u, x, y) \leq 0, \quad \forall y \in K
$$

This completes the proof.

\section{Levitin-Polyak Well-Posedness of $\operatorname{ELP}(F, \phi, K)$}

In this section, we extend the concepts of Levitin-Poylak wellposedness to the equilibrium-like problem and establish its metric characterizations. Let $\alpha \geq 0$ be a given number, and let $X, K, F$, and $\phi$ be defined as the previous section.

Definition 9. A sequence $\left\{x_{n}\right\} \quad \subset \quad X$ is called an LP $\alpha$ approximating sequence for $\operatorname{ELP}(F, \phi, K)$, if there exist $w_{n} \in$ $X$ with $w_{n} \rightarrow 0$ and $0<\epsilon_{n} \rightarrow 0$ such that $x_{n}+w_{n} \in K$ for all $n \in N$ and there exists $u_{n} \in F\left(x_{n}\right)$ such that

$$
\phi\left(u_{n}, x_{n}, y\right) \leq \frac{\alpha}{2}\left\|x_{n}-y\right\|^{2}+\epsilon_{n}, \quad \forall y \in K, n \in N .
$$

If $\alpha_{1}>\alpha_{2} \geq 0$, then every LP $\alpha_{2}$-approximating sequence is LP $\alpha_{1}$-approximating. When $\alpha=0$, we say that $\left\{x_{n}\right\}$ is an $\mathrm{LP}$ approximating sequence for $\operatorname{ELP}(F, \phi, K)$.

Definition 10. $\operatorname{ELP}(F, \phi, K)$ is strongly LP $\alpha$-well-posed if $\operatorname{ELP}(F, \phi, K)$ has an unique solution and every LP $\alpha$ approximating sequence converges strongly to the unique solution. In the sequel, strong LP 0-well-posedness is always called as strong LP well-posedness. If $\alpha_{1}>\alpha_{2} \geq 0$, then strong LP $\alpha_{1}$-well-posedness implies strong LP $\alpha_{2}$-well-posedness.

Definition 11. $\operatorname{ELP}(F, \phi, K)$ is strongly LP $\alpha$-well-posed in the generalized sense if $\operatorname{ELP}(F, \phi, K)$ has nonempty solution set $S$ and every LP $\alpha$-approximating sequence has a subsequence which converges strongly to some point of $S$. In the sequel, strong LP 0-well-posedness in the generalized sense is always called as strong LP well-posedness in the generalized sense. If $\alpha_{1}>\alpha_{2} \geq 0$, then strong LP $\alpha_{1}$-well-posedness in the generalized sense implies strong LP $\alpha_{2}$-well-posedness in the generalized sense.
Remark 12. If $\phi(u, x, y)=\langle u, x-y\rangle+\varphi(x)-\varphi(y)$, for all $x, y \in$ $X, u \in F(x)$, then Definitions 10 and 11 reduce to Definitions 3.3 and 3.4 of [14], respectively. Moreover, when $X$ is a Hilbert space, $K=X$, and $w_{n} \equiv 0$, Definitions 10 and 11 reduce to Definitions 3.2 and 3.3 of [11], respectively.

To obtain the metric characterizations of LP $\alpha$-wellposedness, we consider the following LP $\alpha$-approximating solution set of $\operatorname{ELP}(F, \phi, K)$ :

$$
\begin{aligned}
& \Omega_{\alpha}(\epsilon)=\{x \in \operatorname{dom} \phi: \\
& d(x, K) \leq \epsilon,
\end{aligned}
$$

and there exists $u \in F(x)$

$$
\text { such that } \left.\forall y \in K, \phi(u, x, y) \leq \frac{\alpha}{2}\|x-y\|^{2}+\epsilon\right\} \text {, }
$$

$$
\forall \epsilon \geq 0 \text {. }
$$

Theorem 13. Let $K$ be a nonempty, closed, and convex subset of $X$ and let $F: X \rightarrow 2^{X^{*}}$ be a $\mathscr{H}$-hemicontinuous and nonempty compact-valued mapping. Let $\phi: X^{*} \times X \times X \rightarrow$ $\mathbb{R}$ be monotone with respect to $F$, lower semicontinuous in second argument, and concave in third argument. Moreover, $\phi(u, x, x)=0$, for all $u \in X^{*}, x \in K$. Then, $\operatorname{ELP}(F, \phi, K)$ is strongly LP $\alpha$-well-posed if and only if

$$
\Omega_{\alpha}(\epsilon) \neq \emptyset, \quad \forall \epsilon>0 \text { and } \operatorname{diam}\left(\Omega_{\alpha}(\epsilon)\right) \longrightarrow 0 \quad \text { as } \epsilon \longrightarrow 0 .
$$

Proof. First, we assume that $\operatorname{ELP}(F, \phi, K)$ is strongly $\operatorname{LP} \alpha$ well-posed and $x^{*} \in K$ is the unique solution of $\operatorname{ELP}(F, \phi, K)$. It is easy to see that $x^{*} \in \Omega_{\alpha}(\epsilon)$. If $\operatorname{diam}\left(\Omega_{\alpha}(\epsilon)\right) \nrightarrow 0$ as $\epsilon \rightarrow$ 0 , then there exist constant $l>0$ and sequences $\left\{\epsilon_{n}\right\} \subset \mathbb{R}_{+}$ with $\epsilon_{n} \rightarrow 0$ and $\left\{x_{n}^{(1)}\right\},\left\{x_{n}^{(2)}\right\}$ with $x_{n}^{(1)}, x_{n}^{(2)} \in \Omega_{\alpha}\left(\epsilon_{n}\right)$ such that

$$
\left\|x_{n}^{(1)}-x_{n}^{(2)}\right\|>l, \quad \forall n \in N .
$$

Because of $x_{n}^{(1)}, x_{n}^{(2)} \in \Omega_{\alpha}\left(\epsilon_{n}\right)$, by the definition of $\Omega_{\alpha}\left(\epsilon_{n}\right)$, for $x_{n}^{(1)}$, we obtain

$$
d\left(x_{n}^{(1)}, K\right) \leq \epsilon_{n}<\epsilon_{n}+\frac{1}{n}
$$

and there exists $u_{n} \in F\left(x_{n}^{(1)}\right)$ such that

$$
\phi\left(u_{n}, x_{n}^{(1)}, y\right) \leq \frac{\alpha}{2}\left\|x_{n}^{(1)}-y\right\|^{2}+\epsilon_{n}, \quad \forall y \in K .
$$

Since $K$ is closed and convex, then there exists $\bar{x}_{n}^{(1)} \in K$ such that $\left\|x_{n}^{(1)}-\bar{x}_{n}^{(1)}\right\|<\epsilon_{n}+(1 / n)$. Let $w_{n}=\bar{x}_{n}^{(1)}-x_{n}^{(1)}$; we get $w_{n}+$ $x_{n}^{(1)}=\bar{x}_{n}^{(1)} \in K$ and $\left\|w_{n}\right\|=\left\|x_{n}^{(1)}-\bar{x}_{n}^{(1)}\right\| \rightarrow 0$. This implies that $w_{n} \rightarrow 0$. Thus, $\left\{x_{n}^{(1)}\right\}$ is an LP approximating sequence for $\operatorname{ELP}(F, \phi, K)$. By the similar argument, we obtain that $\left\{x_{n}^{(2)}\right\}$ is an $\operatorname{LP}$ approximating sequence for $\operatorname{ELP}(F, \phi, K)$. So 
they have to converge strongly to the unique solution of $\operatorname{ELP}(F, \phi, K)$, which contradicts condition (13).

Conversely, suppose that condition (12) holds. Let $\left\{x_{n}\right\} \subset$ $X$ be an $\operatorname{LP} \alpha$-approximating sequence for $\operatorname{ELP}(F, \phi, K)$. Then, there exists $w_{n} \in X$ with $w_{n} \rightarrow 0$ such that $x_{n}+w_{n} \in K$, and there exist $0<\epsilon_{n}^{\prime} \rightarrow 0$ and $u_{n} \in F\left(x_{n}\right)$ such that

$$
\phi\left(u_{n}, x_{n}, y\right) \leq \frac{\alpha}{2}\left\|x_{n}-y\right\|^{2}+\epsilon_{n}^{\prime}, \quad \forall y \in K, n \in N .
$$

Since $x_{n}+w_{n} \in K$, then there exists $\bar{x}_{n} \in K$ such that $x_{n}+w_{n}=\bar{x}_{n}$. It is obvious that $d\left(x_{n}, K\right) \leq\left\|x_{n}-\bar{x}_{n}\right\|=$ $\left\|w_{n}\right\| \rightarrow 0$. Suppose that $\epsilon_{n}=\max \left\{\epsilon_{n}^{\prime},\left\|w_{n}\right\|\right\}$; we get that $x_{n} \in$ $\Omega_{\alpha}\left(\epsilon_{n}\right)$. From (12), we have that $\left\{x_{n}\right\}$ is a Cauchy sequence and converges strongly to a point $\bar{x} \in K$. Since $\phi$ is monotone with respect to $F$ and lower semicontinuous in second argument, it follows from (16) that, for any $y \in K, v \in F(y)$,

$$
\begin{aligned}
\phi(v, \bar{x}, y) & \leq \liminf _{n \rightarrow \infty}\left\{\phi\left(v, x_{n}, y\right)\right\} \\
& \leq \liminf _{n \rightarrow \infty}\left\{\phi\left(u_{n}, x_{n}, y\right)\right\} \\
& \leq \liminf _{n \rightarrow \infty}\left\{\frac{\alpha}{2}\left\|x_{n}-y\right\|^{2}+\epsilon_{n}^{\prime}\right\} \\
& =\frac{\alpha}{2}\|\bar{x}-y\|^{2} .
\end{aligned}
$$

For any $y \in K$, let $y_{t}=\bar{x}+t(y-\bar{x})$, for all $t \in[0,1]$. Since $K$ is a nonempty, closed, and convex subset, we have that $y_{t} \in K$. Then, (17) implies that

$$
\phi\left(v_{t}, \bar{x}, y_{t}\right) \leq \frac{\alpha}{2}\left\|\bar{x}-y_{t}\right\|^{2}, \quad \forall v_{t} \in F\left(y_{t}\right) .
$$

Since $\phi$ is concave in third argument and $\phi(u, x, x)=0$, for all $u \in X^{*}, x \in K$,

$$
\phi\left(v_{t}, \bar{x}, y\right) \leq \frac{\alpha t}{2}\|\bar{x}-y\|^{2}, \quad \forall v_{t} \in F\left(y_{t}\right), y \in K .
$$

Since $F$ is a nonempty compact-valued mapping and $\mathscr{H}$ hemicontinuous, by Lemma 2, for each fixed $v_{t} \in F\left(y_{t}\right)$ and each $t \in(0,1)$, there exists a $u_{t} \in F(\bar{x})$ such that $\left\|v_{t}-u_{t}\right\| \leq \mathscr{H}\left(F\left(y_{t}\right), F(\bar{x})\right)$. Since $F$ is $\mathscr{H}$-hemicontinuous, we get that $\left\|v_{t}-u_{t}\right\| \leq \mathscr{H}\left(F\left(y_{t}\right), F(\bar{x})\right) \rightarrow 0$ as $t \rightarrow 0^{+}$. Since $F$ is compact, without loss of generality, we assume that $u_{t} \rightarrow u \in F(\bar{x})$ as $t \rightarrow 0^{+}$. Thus, we obtain that

$$
\begin{aligned}
\left\|v_{t}-u\right\| & \leq\left\|v_{t}-u_{t}\right\|+\left\|u_{t}-u\right\| \\
& \leq \mathscr{H}\left(F\left(y_{t}\right), F(\bar{x})\right)+\left\|u_{t}-u\right\| \longrightarrow 0 \text { as } t \longrightarrow 0^{+} .
\end{aligned}
$$

This implies that $v_{t} \rightarrow u$ as $t \rightarrow 0^{+}$. It follows from (19) that

$$
\phi(u, \bar{x}, y) \leq 0, \quad \forall y \in K .
$$

Therefore, $\bar{x}$ solves $\operatorname{ELP}(F, \phi, K)$.

To complete the proof, we only need to prove that $\operatorname{ELP}(F, \phi, K)$ has a unique solution. Suppose that $\operatorname{ELP}(F, \phi, K)$ has two distinct solutions $x_{1}$ and $x_{2}$. Then, it is obvious that $x_{1}, x_{2} \in \Omega_{\alpha}(\epsilon)$ for all $\epsilon>0$ and

$$
0<\left\|x_{1}-x_{2}\right\| \leq \operatorname{diam}\left(\Omega_{\alpha}(\epsilon)\right) \longrightarrow 0,
$$

a contradiction to (12). This completesthe proof.
Theorem 14. Let $K$ be a nonempty, closed, and convex subset of $X$ and let $F: X \rightarrow 2^{X^{*}}$ be a $\mathscr{H}$-hemicontinuous and nonempty compact-valued mapping. Let $\phi: X^{*} \times X \times X \rightarrow \mathbb{R}$ be monotone with respect to $F$ and lower semicontinuous in second argument. Moreover, $\phi(u, x, x)=0$, for all $u \in X^{*}$, $x \in K$. Then, $\operatorname{ELP}(F, \phi, K)$ is strongly LP $\alpha$-well-posed in the generalized sense if and only if

$$
\Omega_{\alpha}(\epsilon) \neq \emptyset, \quad \forall \epsilon>0 \text { and } \mu\left(\Omega_{\alpha}(\epsilon)\right) \longrightarrow 0 \quad \text { as } \epsilon \longrightarrow 0 .
$$

Proof. Assume that $\operatorname{ELP}(F, \phi, K)$ is strongly $\operatorname{LP} \alpha$-well-posed in the generalized sense. Let $S$ be the solution set of $\operatorname{ELP}(F, \phi, K)$. Then, $S$ is nonempty and compact. Indeed, let $\left\{x_{n}\right\}$ be any sequence in $S$. Then, $\left\{x_{n}\right\}$ is an LP $\alpha$ approximating sequence for $\operatorname{ELP}(F, \phi, K)$. Since $\operatorname{ELP}(F, \phi, K)$ is strongly $\alpha$-well-posed in the generalized sense, $\left\{x_{n}\right\}$ has a subsequence which converges strongly to some point of $S$. Thus, $S$ is compact. It is easy to see that $\Omega_{\alpha}(\epsilon) \supset S \neq \emptyset$ for all $\epsilon>0$. Now we show that

$$
\mu\left(\Omega_{\alpha}(\epsilon)\right) \longrightarrow 0 \quad \text { as } \epsilon \longrightarrow 0 .
$$

It is easy to see that, for every $\epsilon>0$,

$$
\begin{aligned}
\mathscr{H}\left(\Omega_{\alpha}(\epsilon), S\right) & =\max \left\{e\left(\Omega_{\alpha}(\epsilon), S\right), e\left(S, \Omega_{\alpha}(\epsilon)\right)\right\} \\
& =e\left(\Omega_{\alpha}(\epsilon), S\right) .
\end{aligned}
$$

Taking into account the compactness of $S$, we obtain

$$
\mu\left(\Omega_{\alpha}(\epsilon)\right) \leq 2 \mathscr{H}\left(\Omega_{\alpha}(\epsilon), S\right)+\mu(S)=2 e\left(\Omega_{\alpha}(\epsilon), S\right) .
$$

To prove (23), it is sufficient to show that

$$
e\left(\Omega_{\alpha}(\epsilon), S\right) \longrightarrow 0 \quad \text { as } \epsilon \longrightarrow 0 .
$$

Indeed, if $e\left(\Omega_{\alpha}(\epsilon), S\right) \nrightarrow 0$ as $\epsilon \rightarrow 0$, then there exist $l>0$ and $\left\{\epsilon_{n}\right\} \subset \mathbb{R}^{+}$with $\epsilon_{n} \rightarrow 0$, and $x_{n} \in \Omega_{\alpha}\left(\epsilon_{n}\right)$ such that

$$
x_{n} \notin S+B(0, l), \quad \forall n \in N,
$$

where $B(0, l)$ is the closed ball centered at 0 with radius $l$. By the definition of $\Omega_{\alpha}\left(\epsilon_{n}\right)$, we know that $d\left(x_{n}, K\right) \leq \epsilon_{n}<\epsilon_{n}+$ $(1 / n)$, and there exists $u_{n} \in F\left(x_{n}\right)$ such that

$$
\phi\left(u_{n}, x_{n}, y\right) \leq \frac{\alpha}{2}\left\|x_{n}-y\right\|^{2}+\epsilon_{n}, \quad \forall y \in K .
$$

Thus, there exists $\bar{x}_{n} \in K$ such that $\left\|\bar{x}_{n}-x_{n}\right\|<\epsilon_{n}+(1 / n)$. Let $w_{n}=\bar{x}_{n}-x_{n}$; then, we have $w_{n}+x_{n} \in K$ with $w_{n} \rightarrow 0$. So $\left\{x_{n}\right\}$ is an LP $\alpha$-approximating sequence for $\operatorname{ELP}(F, \phi, K)$. Since $\operatorname{ELP}(F, \phi, K)$ is strongly LP $\alpha$-well-posed in the generalized sense, there exists a subsequence $\left\{x_{n_{k}}\right\}$ of $\left\{x_{n}\right\}$ which converges strongly to some point of $S$. This contradicts (28) and so

$$
e\left(\Omega_{\alpha}(\epsilon), S\right) \longrightarrow 0 \quad \text { as } \epsilon \longrightarrow 0 .
$$

Conversely, suppose that (23) holds. We first show that $\Omega_{\alpha}(\epsilon)$ is closed for all $\epsilon>0$. Let $\left\{x_{n}\right\} \subset \Omega_{\alpha}(\epsilon)$ with $x_{n} \rightarrow x$; then, there exists $u_{n} \in F\left(x_{n}\right)$ such that $d\left(x_{n}, K\right) \leq \epsilon$ and

$$
\phi\left(u_{n}, x_{n}, y\right) \leq \frac{\alpha}{2}\left\|x_{n}-y\right\|^{2}+\epsilon, \quad \forall y \in K, n \in N .
$$


Since $F$ is an upper semicontinuous and nonempty compactvalued mapping, there exist a sequence $\left\{u_{n_{k}}\right\}$ of $\left\{u_{n}\right\}$ and some $u \in F(x)$ such that $u_{n_{k}} \rightarrow u$. Therefore, it follows from (31) and the lower semicontinuity of $\phi$ that

$$
\phi(u, x, y) \leq \frac{\alpha}{2}\|x-y\|^{2}+\epsilon, \quad \forall y \in K .
$$

It is obvious that $d(x, K) \leq \epsilon$. This implies that $x \in \Omega_{\alpha}(\epsilon)$ and so $\Omega_{\alpha}(\epsilon)$ is nonempty closed for all $\epsilon>0$. Observe that

$$
S=\bigcap_{\epsilon>0} \Omega_{\alpha}(\epsilon) .
$$

Since $\mu\left(\Omega_{\alpha}(\epsilon)\right) \rightarrow 0$, the theorem in page 412 of [15] can be applied and one concludes that $S$ is nonempty and compact with

$$
e\left(\Omega_{\alpha}(\epsilon), S\right)=\mathscr{H}\left(\Omega_{\alpha}(\epsilon), S\right) \longrightarrow 0 .
$$

Let $\left\{\widehat{x}_{n}\right\} \subset X$ be an LP $\alpha$-approximating sequence for $\operatorname{ELP}(F, \phi, K)$. Then, there exists $w_{n} \in X$ with $w_{n} \rightarrow 0$ such that $\widehat{x}_{n}+w_{n} \in K$, and there exist $\widehat{u}_{n} \in F\left(\widehat{x}_{n}\right)$ and $0<\epsilon_{n}^{\prime} \rightarrow 0$ such that

$$
\phi\left(\widehat{u}_{n}, \widehat{x}_{n}, y\right) \leq \frac{\alpha}{2}\left\|\widehat{x}_{n}-y\right\|^{2}+\epsilon_{n}^{\prime}, \quad \forall y \in K, n \in N .
$$

Since $\widehat{x}_{n}+w_{n} \in K$, then there exists $\bar{x}_{n} \in K$ such that $\widehat{x}_{n}+w_{n}=$ $\bar{x}_{n}$. It follows that

$$
d\left(\widehat{x}_{n}, K\right) \leq\left\|\widehat{x}_{n}-\bar{x}_{n}\right\|=\left\|w_{n}\right\| \longrightarrow 0 .
$$

Set $\epsilon_{n}=\max \left\{\left\|w_{n}\right\|, \epsilon_{n}^{\prime}\right\}$; we get $\widehat{x}_{n} \in \Omega_{\alpha}\left(\epsilon_{n}\right)$. From (23) and the definition of $\Omega_{\alpha}\left(\epsilon_{n}\right)$, we obtain

$$
d\left(\widehat{x}_{n}, S\right) \leq e\left(\Omega_{\alpha}\left(\epsilon_{n}\right), S\right) \longrightarrow 0 .
$$

Since $S$ is compact, there exists $p_{n} \in S$ such that

$$
\left\|p_{n}-\widehat{x}_{n}\right\|=d\left(\widehat{x}_{n}, S\right) \longrightarrow 0 \text {. }
$$

From the compactness of $S$, there exists a subsequence $\left\{p_{n_{k}}\right\}$ of $\left\{p_{n}\right\}$ which converges strongly to $\bar{p} \in S$. Hence, the corresponding subsequence $\left\{\widehat{x}_{n_{k}}\right\}$ of $\left\{\widehat{x}_{n}\right\}$ converges strongly to $\bar{p} \in S$. Thus, $\operatorname{ELP}(F, \phi, K)$ is strongly LP $\alpha$-well-posed in the generalized sense. The proof is complete.

\section{Conditions for Levitin-Polyak Well-Posedness}

In this section, we get some conditions under which the $\operatorname{ELP}(F, \phi, K)$ in Banach spaces is Levitin-Polyak well-posed.

For any $\delta_{0} \geq 0$, we denote $M\left(\delta_{0}\right)=\left\{x \in X: d_{K}(x) \leq \delta_{0}\right\}$. We have the following result.

Theorem 15. Let $K$ be a nonempty, closed, and convex subset of $X$ and let $F: X \rightarrow 2^{X^{*}}$ be a $\mathscr{H}$-hemicontinuous and nonempty compact-valued mapping. Let $\phi: X^{*} \times X \times X \rightarrow$ $\mathbb{R}$ be monotone with respect to $F$, lower semicontinuous in first and second arguments, and concave in third argument. Moreover, $\phi(u, x, x)=0$, for all $u \in X^{*}, x \in K$. If there exists some $\delta_{0}$ with $\delta_{0}>0$ such that $M\left(\delta_{0}\right)$ is compact, then $\operatorname{ELP}(F, \phi, K)$ is strongly LP $\alpha$-well-posed in the generalized sense.
Proof. Let $\left\{x_{n}\right\}$ be an LP approximating sequence for $\operatorname{ELP}(F, \phi, K)$. Then, there exist $0<\epsilon_{n}^{\prime} \rightarrow 0$ and $w_{n} \in X$ with $w_{n} \rightarrow 0$ such that

$$
x_{n}+w_{n} \in K
$$

and there exists $u_{n} \in F\left(x_{n}\right)$ satisfying

$$
\phi\left(u_{n}, x_{n}, y\right) \leq \frac{\alpha}{2}\left\|x_{n}-y\right\|^{2}+\epsilon_{n}^{\prime}, \quad \forall y \in K, n \in N .
$$

Since $x_{n}+w_{n} \in K$, then there exists $\bar{x}_{n} \in K$ such that $x_{n}+w_{n}=$ $\bar{x}_{n}$. Thus,

$$
d\left(x_{n}, K\right) \leq\left\|x_{n}-\bar{x}_{n}\right\|=\left\|w_{n}\right\| \longrightarrow 0 .
$$

Let $\epsilon_{n}=\max \left\{\epsilon_{n}^{\prime},\left\|w_{n}\right\|\right\}$; we can get $d\left(x_{n}, K\right) \leq \epsilon_{n}$. Without loss of generality, suppose that $\left\{x_{n}\right\} \quad \subset M\left(\delta_{0}\right)$ for $n$ is sufficiently large. By the compactness of $M\left(\delta_{0}\right)$, there exist a subsequence $\left\{x_{n_{k}}\right\}$ of $\left\{x_{n}\right\}$ and $\bar{x} \in M\left(\delta_{0}\right)$ such that $x_{n_{k}} \rightarrow \bar{x}$. It is easy to see that $\bar{x} \in K$. Furthermore, by the u.s.c. of $F$ at $\bar{x}$ and compactness of $F(\bar{x})$, there exist a subsequence $\left\{u_{n_{k}}\right\}$ of $\left\{u_{n}\right\}$ and some $\bar{u} \in F(\bar{x})$ such that $u_{n_{k}} \rightarrow \bar{u}$. Since $\phi$ is lower semicontinuous in first and second arguments, it follows from (40) that

$$
\phi(\bar{u}, \bar{x}, y) \leq \frac{\alpha}{2}\|\bar{x}-y\|^{2}, \quad \forall y \in K .
$$

For any $y \in K$, let $y_{t}=\bar{x}+t(y-\bar{x})$, for all $t \in(0,1)$; it is obvious that $y_{t} \in K$. Now, from (42), we have

$$
\phi\left(\bar{u}, \bar{x}, y_{t}\right) \leq \frac{\alpha}{2}\left\|\bar{x}-y_{t}\right\|^{2} .
$$

By the convexity of $\phi$, it follows that, for each $t \in(0,1)$, we obtain

$$
\phi(\bar{u}, \bar{x}, y) \leq \frac{\alpha t}{2}\|\bar{x}-y\|^{2}, \quad \forall y \in K .
$$

Let $t \rightarrow 0^{+}$in the last inequality; then, we have

$$
\phi(\bar{u}, \bar{x}, y) \leq 0, \quad \forall y \in K .
$$

This shows that $\bar{x}$ solves $\operatorname{ELP}(F, \phi, K)$. Thus, $\operatorname{ELP}(F, \phi, K)$ is strongly LP $\alpha$-well-posed in the generalized sense.

\section{Conflict of Interests}

The author declares that there is no conflict of interests regarding the publication of this paper.

\section{References}

[1] A. N. Tykhonov, "On the stability of the functional optimization problem," USSR Computational Mathematics and Mathematical Physics, vol. 6, pp. 631-634, 1966.

[2] T. Zolezzi, "Extended well-posedness of optimization problems," Journal of Optimization Theory and Applications, vol. 91, no. 1, pp. 257-266, 1996.

[3] T. Zolezzi, "Well-posedness criteria in optimization with application to the calculus of variations," Nonlinear Analysis: Theory, Methods and Applications, vol. 25, no. 5, pp. 437-453, 1995. 
[4] T. Zolezzi, "Well-posedness of optimal control problems," Control and Cyber-Netics, vol. 23, pp. 289-301, 1994.

[5] E. S. Levitin and B. T. Polyak, "Convergence of minimizing sequences in conditional extremum problem," Soviet Mathematics Doklady, vol. 7, pp. 764-767, 1996.

[6] E. M. Bednarczuk, "Well-posedness of optimization problem," in Recent Advances and Historical Development of Vector Optimization Problems, J. Jahn and W. Krabs, Eds., vol. 294 of Lecture Notes in Economics and Mathematical Systems, pp. 5161, Springer, Berlin, Germany, 1987.

[7] R. Lucchetti and F. Patrone, "A characterization of Tykhonov well-posedness for minimum problems with applications to variational inequalities," Numerical Functional Analysis and Optimization, vol. 3, no. 4, pp. 461-476, 1981.

[8] R. Lucchetti and F. Patrone, "Hadamard and Tyhonov wellposedness of a certain class of convex functions," Journal of Mathematical Analysis and Applications, vol. 88, no. 1, pp. 204215, 1982.

[9] L. C. Ceng and J. C. Yao, "Well-posedness of generalized mixed variational inequalities, inclusion problems and fixed-point problems," Nonlinear Analysis: Theory, Methods and Applications, vol. 69, no. 12, pp. 4585-4603, 2008.

[10] Y.-P. Fang, N.-J. Huang, and J.-C. Yao, "Well-posedness by perturbations of mixed variational inequalities in Banach spaces," European Journal of Operational Research, vol. 201, no. 3, pp. 682-692, 2010.

[11] Y.-P. Fang, N.-J. Huang, and J.-C. Yao, "Well-posedness of mixed variational inequalities, inclusion problems and fixed point problems," Journal of Global Optimization, vol. 41, no. 1, pp. 117$133,2008$.

[12] B. Lemaire, "conditions, and regularization of minimization, inclusion, and fixed-point problems," Pliska Studia Mathematic Bulgaria, vol. 12, pp. 71-84, 1998.

[13] B. Lemaire, C. Ould Ahmed Salem, and J. P. Revalski, "Wellposedness by perturbations of variational problems," Journal of Optimization Theory and Applications, vol. 115, no. 2, pp. 345368, 2002.

[14] X.-B. Li and F.-Q. Xia, "Levitin-Polyak well-posedness of a generalized mixed variational inequality in Banach spaces," Nonlinear Analysis: Theory, Methods and Applications, vol. 75, no. 4, pp. 2139-2153, 2012.

[15] K. Kuratowski, Topology, vol. 1-2, Academic Press, New York, NY, USA, 1968. 


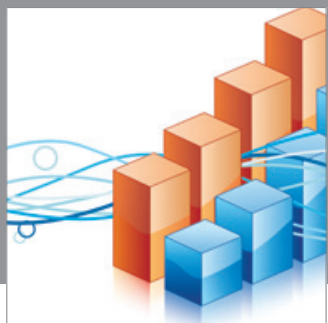

Advances in

Operations Research

mansans

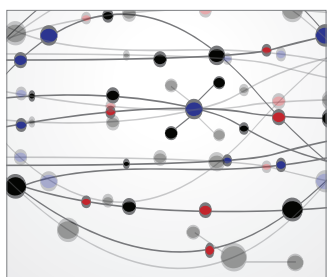

The Scientific World Journal
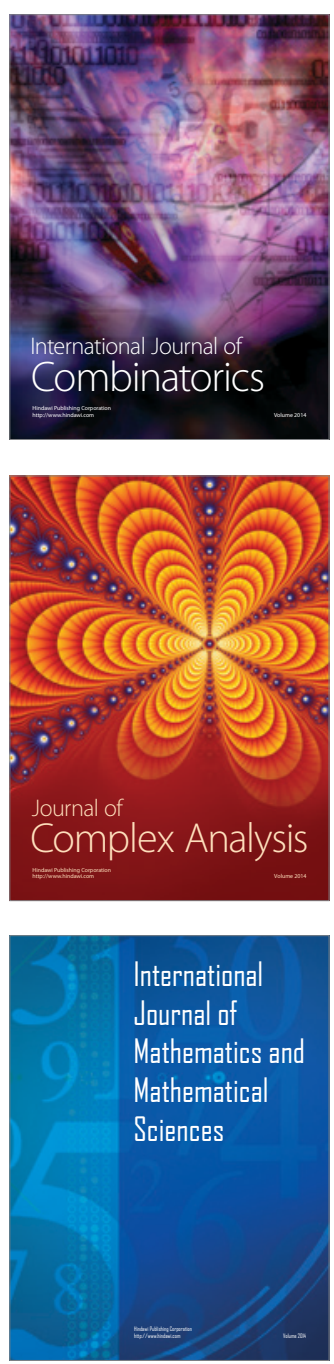
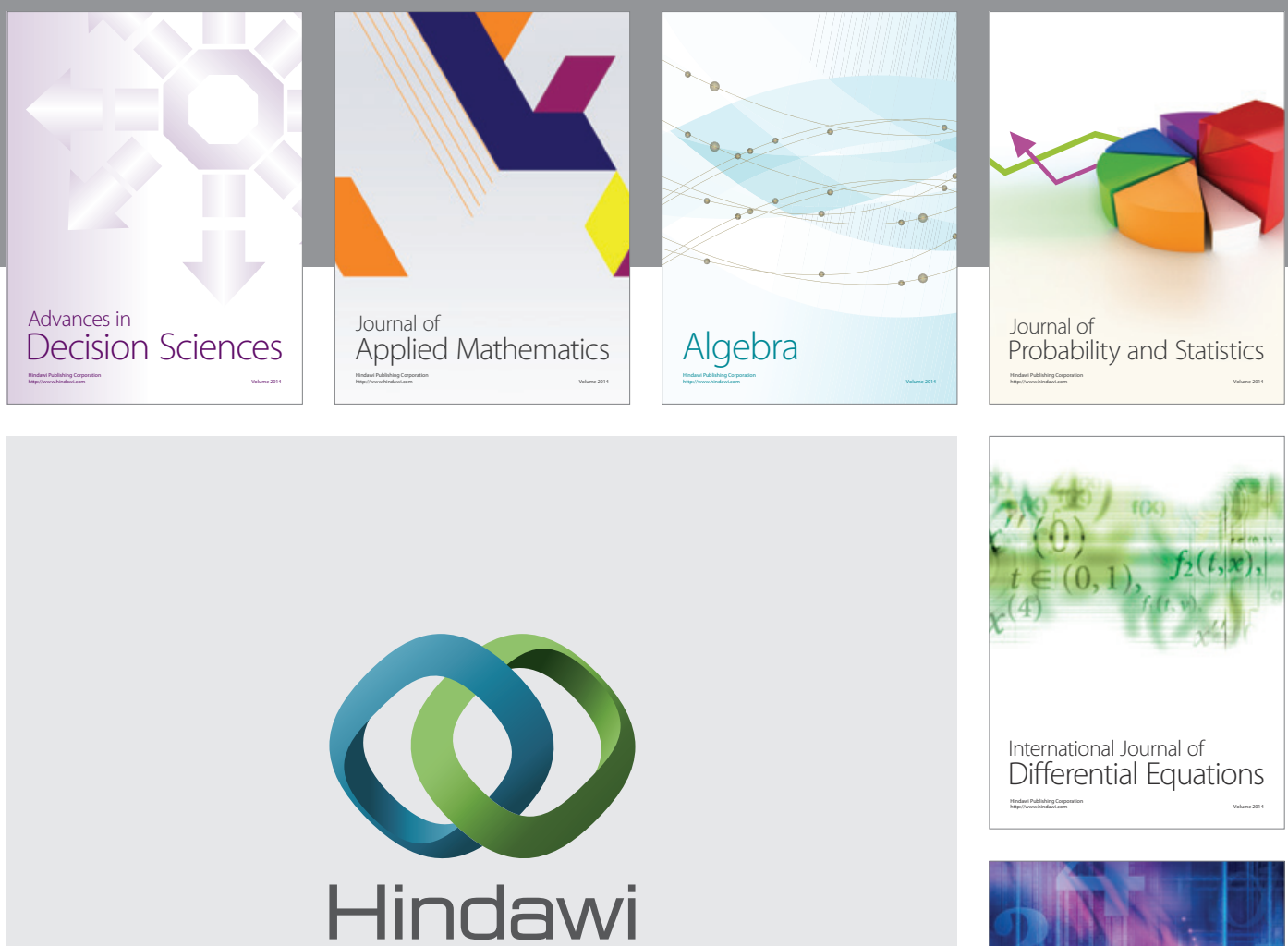

Submit your manuscripts at http://www.hindawi.com
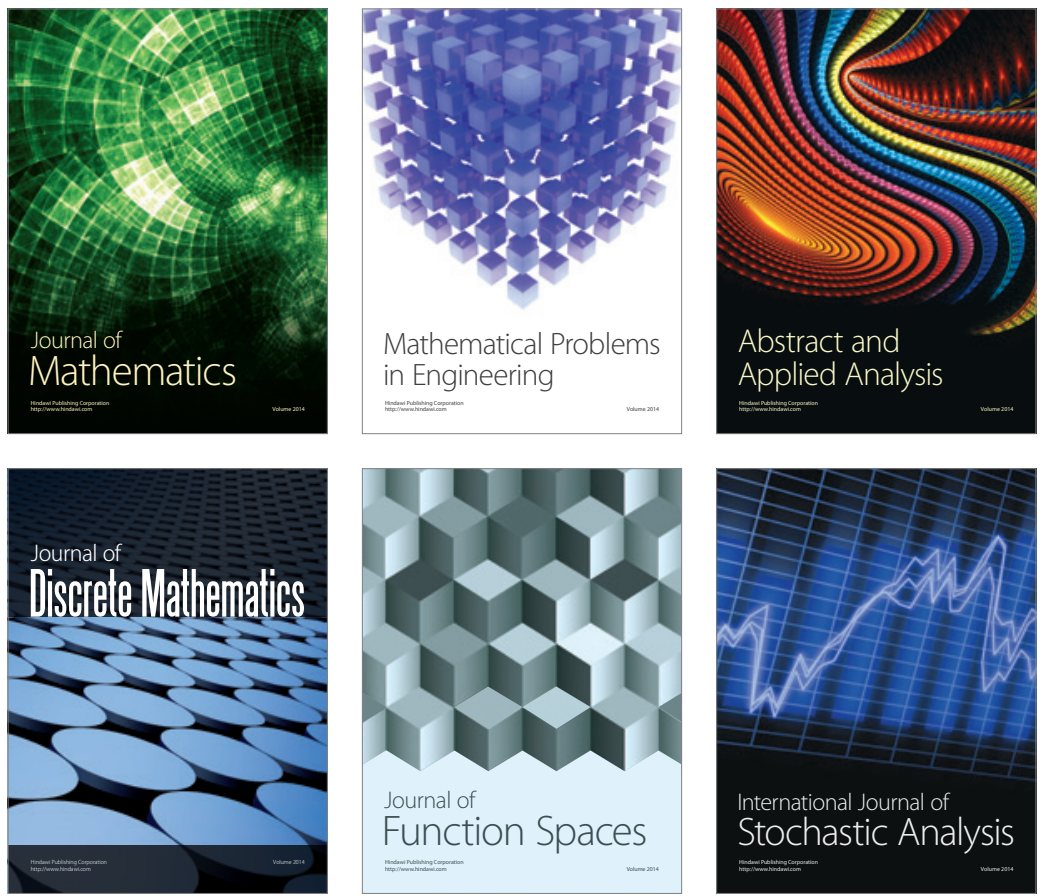

Journal of

Function Spaces

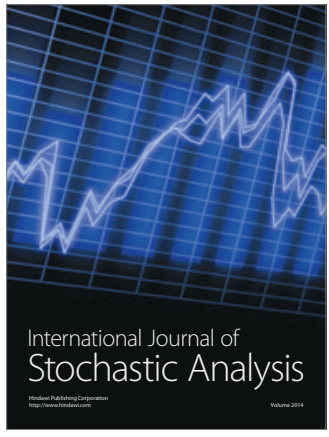

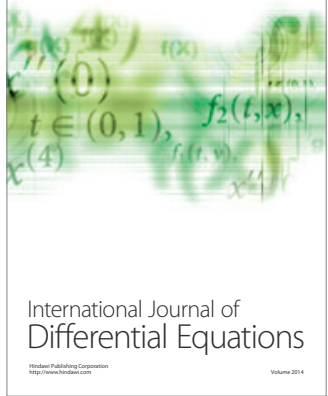
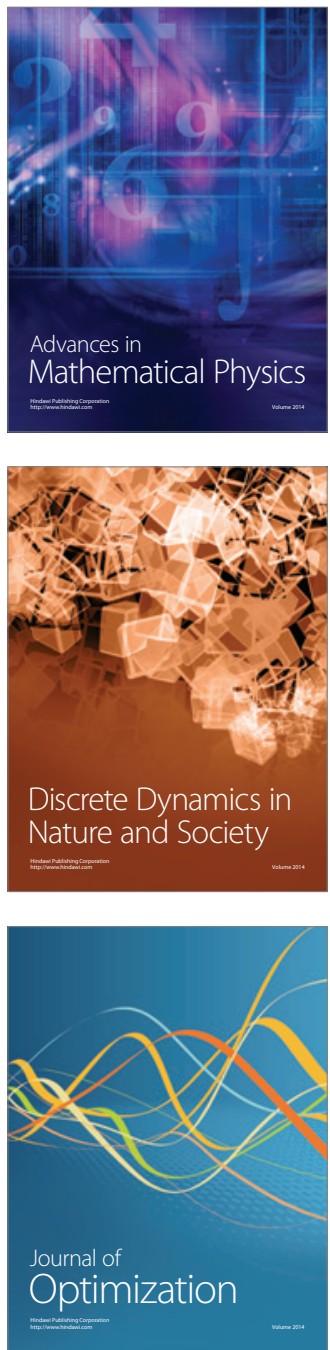